\title{
Structural Equation Model for Evaluating Factors Affecting Health-related Quality of Life in Adolescents With Moyamoya Disease: Based on Salutogenesis Theory
}

Won-oak Oh

Korea University

Insun Yeom ( $\sim$ ruthin76@yuhs.ac)

Severance Hospital https://orcid.org/0000-0001-9914-9486

Sung-Hyun Lim

Korea University

\section{Research}

Keywords: adolescent, chronic disease, health behavior, quality of life, social support, stress, structural equation model

Posted Date: January 4th, 2022

DOI: https://doi.org/10.21203/rs.3.rs-1175504/v1

License: (9) (1) This work is licensed under a Creative Commons Attribution 4.0 International License. Read Full License 


\section{Abstract}

\section{Background}

Moyamoya disease is a cerebrovascular disorder and a significant chronic health concern requiring regular monitoring to control the disease and its related complications.

\section{Objective}

To develop a structural model based on the salutogenesis theory, and to identify how social support, sense of coherence, and stress contribute to health behaviors, subjective health status, and quality of life in adolescents with moyamoya disease.

Methods

We examined a hypothetical model by integrating the concepts of a structural health-related quality-of-life model based on the salutogenesis theory among 239 adolescents with moyamoya disease in Korea. Data on health-related quality of life of adolescents with moyamoya disease were collected using the following scales: social support rating scale, sense of coherence scale, stress scale, health behavior scale, subjective health status scale, and quality-of-life scale. A structural equation model was used to analyze the data.

Results

The final model demonstrated goodness-of-fit. A sense of coherence directly influenced quality of life $(\beta=0.504, p<0.01)$ and indirectly influenced quality of life $(\beta=0.299, p<0.05)$ through health behavior. Stress $(\beta=-0.414, p<0.001)$ and health behavior $(\beta=-0.085, p<0.01)$ directly influenced quality of life. Social support directly influenced health behavior $(\beta=0.321, p<0.01)$ and subjective health status $(\beta=0.112, p<0.01)$.

\section{Discussion}

Since moyamoya disease, a chronic disease, is progressive, it is very important to identify factors for health promotion. This study found that sense of coherence and social support were significant factors contributing to lower stress, improved health, and quality of life in adolescents with moyamoya disease. This paper is intended to help health experts to develop an intervention strategy based on theory as an approach for chronic disease management.

\section{Background}

With the development of modern medical technology, research on rare and incurable diseases is conducted more regularly. For this reason, we can now monitor rare, incurable, and chronic diseases on a daily basis. Moyamoya disease, a rare and incurable disease, is a primary cause of strokes or transient ischemic attacks in adolescents [1]. Incidences of moyamoya disease in adolescents have increased in recent years, requiring continuous management despite surgical treatments [2].

Transient ischemic attacks affect approximately $70 \%$ of adolescents with moyamoya disease and symptoms such as headaches, hemiparesis, and seizures may occur temporarily or permanently $[3,4,5]$. Decreased brain perfusion can cause cognitive impairments, intellectual decline, and mental retardation [2].

To prevent symptoms that cause severe sequelae, reducing risk factors and managing symptom triggers is essential. In terms of disease management, it is important to identify the health behaviors required for patients to manage moyamoya disease and the degree to which they must be implemented. Moyamoya disease can easily cause cerebral vascular hypo circulation-related symptoms even with minor changes in blood flow. It can also cause transient cerebral ischemic attacks in stressful situations [6]; therefore, stress management is required [7, 8]. The health issues and symptoms experienced by adolescents with moyamoya disease are often stress-related [9]. Therefore, it is necessary to understand the relationship between stress and health in adolescents with moyamoya disease to prevent the exacerbation of these problems and provide age-appropriate stress and healthmanagement interventions. 
Few studies have explored the promotion of healthy behaviors among adolescents with moyamoya disease in-depth. Therefore, to promote the health of these adolescents, it is important first to understand the typical health status of moyamoya disease patients and identify factors affecting their health and quality of life.

According to the Bangkok Charter adopted at the Sixth Global Conference on Health Promotion, "Health promotion is the process by which a person develops the ability to control his or her health and its determinants, thereby becoming healthier" [10]. This means that an individual's organizational and social skills should be nurtured, and appropriate resources provided to identify health problems and find the best solution for them. Therefore, it is necessary to provide a fresh perspective and construct a new concept of health status for adolescents with moyamoya disease.

The salutogenesis theory emphasizes the importance of individual ability and resources to nurture health rather than reducing external stressors. Sense of coherence has been suggested as a critical factor in controlling stress [11].

A previous study applying the salutogenesis theory reported that stress symptoms were reduced [12], health behaviors were well performed, and overall health was good [13]. The salutogenesis theory was integrated with this study, and it seemed a suitable model for promoting health in adolescents with moyamoya disease. This theory emphasizes the individual's ability to use sense of coherence and manage within a stressful environment. It explains the problem-solving process in greater detail so that the individual may more easily adopt health-promoting behaviors.

Adolescents with moyamoya disease are more stressed than other adolescents due to their disease and other developmental challenges, triggering adverse health conditions and symptoms. Sense of coherence and social support have been shown to assist in recovery and promote health by improving an individual's ability to cope with stress as reported in studies of adolescents with other chronic diseases [14]. These results indicate that it is necessary to include social support in the model.

Therefore, this study uses the salutogenesis theory to investigate whether the core factors-sense of coherence, stress, social support, health behaviors, health status, and quality of life-affect the health of adolescents with chronic diseases. Our objective was to build a structural model of health in adolescents with moyamoya disease.

\section{Methods}

\section{Aims}

To develop a structural model based on the salutogenesis theory to identify how social support, sense of coherence, and stress contribute to health behavior, subjective health status, and quality of life in adolescents with moyamoya disease, and to provide primary data for the preparation of systematic interventions.

\section{Design}

This was a single center, cross-sectional study based on the Salutogenesis theory. This study used a structural equation model to suggest and verify a hypothetical model for the factors influencing health-related quality of life in adolescents with moyamoya disease, based on the salutogenesis theory (Antonovsky 1979).

\section{Conceptual Framework and Hypothetical Model}

The conceptual framework of this study is based on Antonovsky's salutogenesis theory [15]. In the theory of health creation, humans are open systems that actively interact with the environment. Interaction with the external environment can be a potential source of disease in stressful situations where people are ill, but it can also be a resource to promote health. Despite the inevitable stress and illness experienced in an individual's life, the theory explains that recovery is possible by actively overcoming stress through the internal abilities and resources of the individual. In other words, essential factors of the health promotion process are generalized resistance resources and sense of coherence [11]. Therefore, we hypothesized that knowledge of moyamoya disease, social support, and sense of coherence affect individual stress and health behaviors, affecting subjective health status, depression, and quality of life (Figure 1).

Based on these path settings, a hypothetical path was constructed, as shown in Figure 1. 


\section{Participants}

The sample included 239 adolescents with moyamoya disease, aged 13-18 years, in the moyamoya clinic at Severance Hospital in South Korea. They had been afflicted by moyamoya disease for over one month, had no other diseases, and were able to respond to a questionnaire. In addition, the exclusion criteria for adolescents with moyamoya disease were a history of mental illness or difficulty in participating (e.g., hearing and visual impairment). All participants confirmed their understanding of the purpose of the study and agreed to participate voluntarily through online written consent.

In general, between two and four hundred samples should be included when applying the maximum likelihood estimation for structural equation modeling [16]. Based on this recommendation, the survey was distributed to 250 participants (considering a $20 \%$ dropout rate). The final number of participants was 239, which excluded 11 participants with insufficient questionnaire responses.

\section{Data collection}

Data were collected after approval by the Severance Hospital Institutional Review Board (4-2017-0884). All procedures performed in this study were in accordance with the ethical standards of the institutional or national research committee. Data were collected face-to-face between January and October of 2018.

Participants were assured of the anonymity and confidentiality of their responses and that any data collected would be used for research purposes only. They were told that they could withdraw from the study at any time. Those who agreed to participate answered the questions. After the survey, the participants each received a gift from the researcher for completing the survey.

\section{Measurement instruments}

\section{Social support}

A translated child and adolescent social support scale [17] was completed by each participant. The self-reported questionnaire consisted of 48 items and was scored on a 6-point Likert scale. Support was assessed on four subscales: support from parents (12 items), support from teachers (12 items), support from classmates (12 items), and support from close friends (12 items). Higher scores indicated more social support in each area. Cronbach's a value was .96 .

\section{Sense of coherence}

The sense of coherence scale [11] was used to measure each participant's sense of coherence. The author translated the tool into Korean and then re-translated it back to English to ensure accuracy. The scale was developed using two tools; the original tool measured 29 items, and the shortened tool measured 13 items. Because the reliability and validity of the shortened tool were verified, this study used the shortened tool for convenience.

The self-reported questionnaire consisted of 13 items and was scored on a 7-point scale, making the score range 13-91. Three sections were measured: comprehensibility (5 items), manageability (4 items), and meaningfulness (4 items). Higher scores indicated a higher sense of coherence. Cronbach's a value was .88.

\section{Health behavior}

The moyamoya health behavior scale (developed by the author) was used to determine the health behavior of adolescents with moyamoya disease (Oh et al. 2021). The self-reported questionnaire consisted of 12 items and was scored on a 5-point scale; therefore, the score range was $12-60$. Three sections were measured: treatment instructions (4 items), health promotion acts (4 items), and health coping behavior (4 items). Higher scores indicated better health behaviors related to moyamoya disease. When the moyamoya health behavior scale was developed, Cronbach's a was .86; in this study, it was .87 .

\section{Stress}

Stress was assessed using the stress scale for adolescents [18]. The scale consists of three subscales measuring physical, behavioral, and emotional stress. All items are scored on a 4-point asymmetrical rating scale, ranging from 1 (not at all) to 4 (a lot). 
There were 27 questions, and the higher the score for each sub-factor, the more stress was experienced. At the time of the tool's development, Cronbach's a was 0.82 ; in this study, it was 0.95 .

\section{Subjective health status}

Subjective health status was a subjective evaluation of the perceived health of the participants. This study consisted of a 5-point simple-question Likert scale, ranging from 1 point (very bad) to 5 points (very good).

\section{Quality of life}

Quality of life assesses subjective satisfaction with the academic functions and physical, mental, and social well-being that one perceives in one's life. In this study, the quality of life of adolescents with moyamoya disease was defined as a measure of the quality of life of adolescents (ages 13 to 18) developed by Varni et al. [19].

\section{Ethical considerations}

After receiving approval from the IRB of the investigator's affiliated hospital (4-2017-0884), subjects were recruited and data were collected. Patients and patient's parent interested in the study were provided with detailed paper instructions. Subjects who voluntarily indicated their intention to participate in the study provided written informed consent.

\section{Data Analysis}

We analyzed the data using IBM SPSS $®$ version 26.0 and AMOS version 25.0. The general characteristics and study variables were summarized using descriptive statistics. Factor loading, average variance extraction, and critical ratio were utilized for the convergent validity of the sample. The discriminant validity between variables was determined using the correlation coefficient $(r)$ and the average variance extracted; statistical data on skewness and kurtosis were obtained for the normality of the samples. Multicollinearity between variables was determined using variance inflation factor and tolerance. A confirmatory factor analysis was performed on the model to evaluate whether the measurement for the latent variable was executed correctly. The goodness-offit of the measurement model, hypothetical model, and modified model was evaluated using $\chi^{2}, \chi^{2} / \mathrm{df}$, adjusted goodness-of-fit index, goodness-of-fit index, comparative fit index, root mean square error of approximation, and standardized root mean squared residual.

We used standard regression weights, critical ratios, $p$ values, and squared multiple correlations to verify the statistical significance of the modified model. The bootstrap maximum estimator was used 10,000 times within the $95 \%$ confidence interval to test the significance of the modified model's direct, indirect, and total effects.

\section{Results}

\section{Participants' general characteristics and key variables related to study}

The participants who responded were $49 \%$ male and $51 \%$ female, with an average age of 14.9 . They were confirmed as having moyamoya disease symptoms (convulsions, ischemic attack, etc.) while at a hospital (93.7\%), during a medical examination $(2.5 \%)$, or during an examination regarding another disease (3.8\%). The duration since diagnosis was less than one year (16.7\%), 1 to 3 years $(31.8 \%)$, and $>3$ years $(51.5 \%)$. Finally, $20.9 \%$ had been educated about the disease before the study (Table 1$)$.

The average scores for the participants' social support was 163.6, cohesion was 61.78 , stress was 43.71 , moyamoya disease health behavior was 41.13 , and quality of life was 92.43 .

Before the structural model analysis, skewness and kurtosis were investigated to check whether the variables were normally distributed. Results showed that skewness was confirmed as -4 to 8.48 , and kurtosis as -1.69 to 5.35 .

When the values of standardized skewness and kurtosis are greater than the absolute value of 3 , it is sometimes judged that a normal distribution was not followed [20].

However, it does not exactly follow the normal distribution according to the central limit theorem. Nevertheless, when the sample size $n$ is large enough, it approximately follows the normal distribution [21]. The value of $n$ in this study was 239 and is, therefore, 
assumed to follow a normal distribution.

Confirmatory factor analysis was performed to evaluate the validity of the constituent factors. The standardized factor loading should be at least .5 and less than .9 , and our results were in that range (.73-.94). We checked the conceptual reliability and the average variance extracted values to verify the concentration validity. We found that the concept reliability value was .7 or higher (.76 .88), and the average variance extracted value was .5 or higher (.62 .72). Additionally, the correlation between the correlation matrix and the average variance extracted was checked to confirm the discriminant validity between the main variables. By comparing the variance extraction index and multiple correlation index of the study's main variables, we found the correlation coefficient between the latent variables of the measurement model for the participants to be in the range of .61 .75. Since the absolute values of the correlation coefficients were all less than .85 , the value of the multiple correlation coefficient was small, confirming that the discriminant validity between factors was secured [16].

\section{Analysis of Structural Model}

\section{Verification of fit for the hypothetical and revised models}

The reliability and validity of each latent variable observed was confirmed through confirmatory factor analysis to verify the model's fit in this study. The fit of the measurement model composed of the confirmed latent variables was verified.

The fit of the initial model of this study was $\chi^{2}=469.359(<.001), \chi^{2} / \mathrm{dt}=4.47$, goodness-of-fit index $=.814$, adjusted goodness-tofit index $=.729$, comparative fit index $=.854$, normed fit index $=.821$, incremental fit index $=.855$, standardized root mean squared residual $=.075$, root mean square error of approximation $=.121$. This indicates that correction is necessary to improve the fit of the model.

During confirmatory factor analysis, it was confirmed that Cronbach's a coefficient for moyamoya health behavior was low when divided by each observation variable (Cronbach's $a=.578-788$ ). Upon confirming that Cronbach's a coefficient improved when the observed variables were grouped (Cronbach's $a=.856$ ), the observed variables of the moyamoya health behavior (treatment instructions, health coping behavior, and health promotion acts) were linked and modified.

The fit of the model could be improved when the model was modified by linking the variables with high correlation coefficients between observed variables (and with a correction index of 10 or more) in stages [22]. Based on this, the model was modified by linking the covariance of the observed variable (teacher-close friend) in the corresponding social support variable with the observed variable (interpersonal relationship function-school function) in the quality-of-life variable.

The final model fit index of the hypothetical model in this study was $\chi^{2}=211.446(<.001), \chi^{2} / \mathrm{dt}=4.066$, goodness-of-fit index $=$ .887 , adjusted goodness-to-fit index $=.803$, comparative fit index $=.910$, normed fit index $=.885$, incremental fit index $=$ .911 , standardized root mean squared residual $=.052$, root mean square error of approximation $=.114$. This confirms that the fitness index increased.

The fit of comparative fit index and incremental fit index confirms that the optimal model criterion is satisfied. Based on the goodness-of-fit index, normed fit index, and standardized root mean squared residual values, the hypothetical model for the health of adolescents with moyamoya disease based on the salutogenesis theory was judged to be suitable, as seen in Table 4.

\section{Estimating the path coefficient for a hypothetical model}

As a result of the hypothetical model analysis, seven out of fourteen pathways in the hypothetical structural model were statistically significant as seen in Table 5 below.

Social support was an insignificant path for stress $(\beta=.052, p=.688)$ and a significant path for sense of coherence $(\beta=-0.373, p$ $=.003)$. The explanatory power of the variables influencing the stress variable was $1.13 \%$.

Social support for moyamoya health behavior $(\beta=.325, p=.005)$ and stress $(\beta=-.131, p=.042)$ were found to be significant pathways, while sense of coherence $(\beta=.178, p=.119)$ was an insignificant path. The explanatory power of the variables affecting the moyamoya health behavior was $27.4 \%$. 
Moyamoya disease health behavior $(\beta=.032, p=.636)$, stress $(\beta=.015, p=.809)$, and social support $(\beta=.084, p=.469)$ were not significant pathways for subjective health status while sense of coherence $(\beta=.467, p<.001)$ was a significant pathway. The explanatory power of the variables affecting subjective health status was $29.5 \%$. Subjective health status $(\beta=.108, p=.064)$ and social support ( $\beta=.137, p=.171$ ) for quality of life were not found to be insignificant.

Moyamoya health behavior $(\beta=-.173, p=.003)$, stress $(\beta=-.557, p<.001)$, and sense of coherence $(\beta=.371, p<.001)$ were significant pathways for quality of life. The explanatory power of the variables affecting quality of life was $68.9 \%$. The path of the hypothetical model for the above results is shown in Figure 2.

\subsubsection{Effects of the modified model}

Table 6 shows the direct, indirect, and total effects of the endogenous variables in the modified model. Sense of coherence had a significant direct effect $(\beta=-.373, p=.004)$ on the stress of moyamoya disease adolescents. Social support $(\beta=.325, p=.006)$ and stress $(\beta=-.131, p=.043)$ had significant direct and total effects on the health behaviors of adolescents with moyamoya disease, and sense of coherence $(\beta=.178, p=.165)$ had a significant indirect effect on the health behaviors of adolescents with moyamoya disease. Only the sense of coherence $(\beta=.467, p<.001)$ had significant direct and total effects on subjective health status.

Health behavior $(\beta=-.173, p=.467)$ and stress $(\beta=-.557, p<.001)$ had significant direct and total effects on the quality of life of adolescents with moyamoya disease. Sense of coherence had a significant direct $(\beta=.371, p=.003)$, indirect $(\beta=.220, p=.013)$, and total $(\beta=.590, p<.001)$ effect on the quality of life of adolescents with moyamoya disease as seen in Table 6 .

\section{Discussion}

This study established the first health status model for adolescents with moyamoya disease based on the salutogenesis theory and is meaningful in that it examines their health behaviors and status. These health-related indicators include knowledge of the disease, social support, and sense of coherence. The factor in this model that had the most significant influence on the health status of adolescents with moyamoya disease was stress. This supports the salutogenesis theory that successful stress management influences an individual's health status [11].

In this study, the stress measurement of adolescents with moyamoya disease was 43.71 points, higher than that of the general adolescent population at 42.50 points on the stress scale for adolescents. This score indicates that adolescents with moyamoya disease fall into the high-risk group for stress among South Korean adolescents [18]. Probable causes for the increased stress in this group include everyday limitations and psychological tension or pressure caused by the disease, and regular medical treatment and examination [2]. Further analysis indicates that the stress of the adolescents in this study was particularly high in the physical domain, followed by the emotional and behavioral domains. These results mirror those of a stress-related survey on adolescents with chronic diseases, in which physical discomfort and limitations due to health problems were some of the leading causes of stress [23].

Sense of coherence had the most significant influence as a variable explaining the stress of adolescents with moyamoya disease. This supports the results of previous studies where adolescents with a high sense of coherence maintained good health and managed stressful situations well $[11,13,24]$. According to the salutogenesis theory, people with a high sense of coherence are more flexible and use adaptive strategies to cope with specific situations and to meet their needs [11]. The sense of coherence refers to managing personal tension, identifying internal and external resources, and mobilizing them to find solutions to effective stress management. A previous study on adolescents with chronic heart disease showed that the group with a high sense of coherence had a lower degree of exhaustion $[25,26]$. Furthermore, a report showed a negative correlation between the sense of coherence and stress in epileptic adolescents, which supports the results of this study [27].

Our study found that sense of coherence directly affected subjective health status and had a significant indirect effect on health behavior. This supports the results of previous studies that show that sense of coherence had a significant correlation with health status $[28,29]$ and promoted health behaviors [31, 31]. 
The indirect effect of moyamoya disease on health behavior (treatment instructions, health coping behavior, health promotion acts) in this study indicates that, as in previous studies, sense of coherence helps with lifestyle modification [32].

The structural model showed that social support had a significant direct effect on health behavior and lowered stress even more than sense of coherence. This supports the results of previous studies that reported positive coping and stress reduction through family and social support systems [33,34,35]. Moyamoya health behavior and stress directly affect the quality of life of adolescents with the disease.

This indicates that the quality of life of adolescents with chronic diseases is higher in groups with good individual health behaviors and the ability to cope with stress [36,37]. On the other hand, sense of coherence has both a direct and indirect effect on quality of life. Previous studies that applied the salutogenesis theory found that the higher the sense of coherence, the fewer stress symptoms there were and the better the individual's quality of life [38,39]. Higher sense of coherence was an indicator of better health behaviors [28] and improved the quality of life. Therefore, sense of coherence is a significant factor affecting quality of life [11].

The model analysis in this study showed that sense of coherence is a predictor of stress, health behavior, subjective health status, and quality of life in adolescents with moyamoya disease. It can therefore be said to be a model supporting the salutogenesis theory. Health promotion based on the salutogenesis theory refers to a process in which adolescents with chronic diseases become the participants themselves, identify the causes of stress in their lives, and use appropriate resources to address them. It is necessary to provide health resources, social support, and interventions that enhance sense of coherence to improve the health of adolescents with moyamoya disease. As they are more likely to be stressed due to their affliction, it is necessary to consider developing a program to promote health and improve their sense of coherence.

Sense of coherence develops when the norms and values of life are apparent, when there are positive experiences of success, when people have access to social resources, and when they are respected in the decision-making process. Lack of social relationships, negative life experiences, and lack of education can hinder the development of sense of coherence [40, 41]. Therefore, to improve the sense of coherence of adolescents with moyamoya disease, it is necessary to promote good health behaviors and activities, provide positive reinforcement when implementing them, and plan interventions that connect them to health provider networks.

\section{Limitations}

Several limitations should be considered when interpreting the results of this study. First, this study is based on a cross-sectional sample. Longitudinal samples are required to understand long-term observational studies on the sense of coherence and health status of adolescents with moyamoya disease. Second, this study was based on the adolescents' subjective interpretation of each item in the questionnaire. Lastly, the results of this study are limited because it was only conducted on adolescents with moyamoya disease at a single hospital in Seoul, South Korea.

\section{Conclusions}

The purpose of this study was to establish a structural model based on Antonovsky's salutogenesis theory to identify how various influencing factors contribute to the health status of adolescents with moyamoya disease.

Antonovsky's salutogenesis theory is a model that emphasizes the individuals' capacity to deal with their health problems. It can be the basis of a health promotion model applied to adolescents coping with stress due to their chronic diseases.

This study found that sense of coherence and social support were major influencing factors in lowering stress and improving the health status and quality of life in adolescents with moyamoya disease.

Sense of coherence is the core of the salutogenesis theory. The understanding thereof is crucial in developing programs and interventions that can help adolescents with moyamoya disease improve their own sense of coherence and health behaviors.

\section{RELEVANCE TO CLINICAL PRACTICE}

Page 8/18 
Since moyamoya disease, a chronic disease, is progressive, it is very important to identify factors for health promotion. The findings from this study have the potential to help nurses to promote the health of adolescents with moyamoya disease and other chronic diseases. Sense of coherence and social support were major influencing factors in lowering stress and improving the health status and quality of life in adolescents with moyamoya disease.

We recommend that nurses monitor the symptoms of adolescents with moyamoya disease, explore how the patients minimize their symptoms and how confident they feel about managing their symptoms-particularly stress and healthy behaviors.

Also, this paper is intended to help health experts to develop an intervention strategy based on theory as an approach for chronic disease management

\section{Declarations}

\section{Ethics approval and consent to participate}

This study was conducted after receiving the Institutional Review Board approval from Human Research Protection Center in the Yonsei University Health System (4-2018-0706) in Seoul, South Korea.

\section{Consent for publication}

Not applicable

\section{Availability of data and materials}

Please contact author for data requests

\section{Funding}

This study was funded by the National Research Foundation of South Korea (grant number NRF-2019R1AC1004633).

\section{Competing interests}

The authors declare that they have no competing interests

\section{Authors' contributions}

Regarding the authors' contributions, Won-oak Oh and Sung-Hyun Lim contributed to the design of the work and the acquisition, analysis and interpretation of data. Insun Yeom contributed to the design of the work; the acquisition, analysis and interpretation of data; drafted the work or substantively revised it; and created new software used in the work.

\section{Acknowledgements}

Not applicable

\section{References}

1. Lee, J.W., Yang, W.S., Chung, S.Y., Kang, J.H., Cho, B., Kim, H.K., Kim, K. M.,Jeong, D.C. 2007. Aggressive Systemic Mastocytosis After Germ Cell Tumor of the Ovary. Journal of Pediatric Hematology, 29(6), 412-415.

2. Kim, J.S. 2016. Moyamoya disease: Epidemiology, clinical features, and diagnosis. Journal of Stroke, 18(1), 2-11.

3. Bang, O., Fujimura, M, Kimc, S.K. 2016. The pathophysiology of moyamoya disease. An update. Journal of Stroke,18(1), 1220.

4. Bersano, A., Guey, S., Bedini, G., Nava, S., Hervé, D., Vajkoczy, P., Klijn, C. 2016. Research Progresses in Understanding the Pathophysiology of Moyamoya Disease. Cerebrovascular Diseases, 41(34), 105-118. 
5. Oh, W.O., Yeom, I.S., Lim, S.H., Kim, D.S., Shim, K.W., 2021. The moyamoya health behavior scale for adolescent patients: Measurement tool development and psychometric evaluation. International Journal of Environmental Research and Public Health, 18(8),1-15.

6. Cho, B.K., Tominaga, T., editors. 2010. Moyamoya Disease Update. 1st ed. Tokyo: Springer Japan.

7. Seo E.K. 2013. Diagnosis and treatment of moyamoya disease. The Ewha Medical Journal, 36(1), 9-17.

8. Kim, M., Park, W., Chung Y., Lee SU, Park J.C., Kwon D.H., Ahn, J.S., Lee, A. 2020. Development and validation of a risk scoring model for postoperative adult moyamoya disease. Journal of Neurosurgery, 134(5), 1505-1514.

9. Mohamed, N., Iqbal, A. 2013. Pediatric Moyamoya disease. American Journal of Case Reports, 14, 134-138.

10. WHO. 2005. The Bangkok Charter for Health Promotion, 'Policy and Partnerships for Acation'. The 6th Global Conference on Health Promotion. 7 -11 August, Bangkok, Thailand.

http://www.who.int/healthpromotion/conferences/6gchp/bangkok_charter (last accessed 22 October 2006).

11. Antonovsky, A. 1987. Unraveling the mystery of health: How people manage stress and stay well. San Francisco: Jossey-Bass.

12. Orna, B.L., Claude-H.M. 2020. Salutogenesis and coping: Ways to overcome stress and conflict. International Journal of Environmental Research and Public Health, 17, 6667.

13. Moksnes, U.K., Byrne, D.G., Espnes, G.A. 2020. Sense of coherence in association with stress experience and health in adolescents. International Journal of Environmental Research and Public Health, 17(9), 3003

14. Abu-Kaf, S., Khalaf, E. 2020. Acculturative stress among Arab students in Israel: The roles of sense of coherence and coping strategies. International Journal of Environmental Research and Public Health, 17, 5106.

15. Antonovsky, A. 1979. Health, stress and coping. San Francisco: Jossey-Bass

16. Woo, J.P. 2016. AMOS and SPSS. Seoul: Hanarea academy. 2016, 564-567.

17. Malecki, C.K., Demaray, M.K., Elliott, S.N., 2000. The child and adolescent social support scale. DeKalb: Northern Illinois University: DeKalb, IL.

18. Kim, B.S., Bae, S.M., 2014. Development and validation of daily stress response scale for adolescents, Journal of Korean Psychology, 11(1), 19-32.

19. Varni, J.W., Katz, E.R., Seid, M., Quiggins, D.J.L., Friedman-Bender, A., 1998. The pediatric cancer quality of life inventory-32 (PCQL-32): I. Reliability and validity. Cancer, 82, 1184-1196.

20. Kang, H. 2013. A guide on the use of factor analysis in the assessment of construct validity. Journal of Korean Academy of Nursing, 43(5), 587-594.

21. Kwak, S.K., Kim, J.H. 2017. Statistical data preparation: Management of missing values and outliers. Korean Journal Anesthesiol, 70, 407.

22. Shin. 2013. Statistical methods with applications to demography and Life Insurance by KHMALADZE, ESTATE V. Biometrics, 69(4), 1087-1088.

23. Freedenberg, V.A., Hinds, P.S., Friedmann, E. 2017. Mindfulness-based stress reduction and group support decrease stress in adolescents with cardiac diagnoses: a randomized two-group study. Pediatric Cardiology, 38, 1415-1425.

24. Moksnes, U.K., Espnes, G.A., Haugan, G. 2013. Stress, sense of coherence, and emotional symptoms in adolescents. Psychology \& Health, 29(1), 32-49.

25. Apers, S., Moons, P., Goossens, E., Luyckx, K., Gewillig, M., Bogaerts, K., Budts, W. 2013. Sense of coherence and perceived physical health explain the better quality of life in adolescents with congenital heart disease. European Journal of Cardiovascular Nursing, 12(5), 475-483

26. Neuner, B., Busch, M.A., Singer, S., Moons, P., Wellmann, J., Bauer, U., Nowak-Gottl, U., Hense, H.W. 2011. Sense of coherence as a predictor of quality of life in adolescents with congenital heart defects: A register-based 1-year follow-up study. Journal of Developmental \& Behavioral Pediatrics, 32(4), 316-327.

27. Gauffin, H., Landtblom, A.M., Räty, L. 2010. Self-esteem and sense of coherence in young people with uncomplicated epilepsy: A 5-year follow-up. Epilepsy \& Behavior, 17(4), 520-524.

28. Lansimiles, H., Pietilä, Anna-Maija., Hietasola-Husu, S., Kangasniemi, M.D. 2017. A systematic review of adolescents' sense of coherence and health Scand. Journal Caring Sciences, 31, 651-661.

Page $10 / 18$ 
29. Apers, A., Luyckx, L., Rassart, J., Goossens, E., Budts, W., Moons, P. 2013. Sense of coherence is a predictor of perceived health in adolescents with congenital heart disease: A cross-lagged prospective study. International Journal of Nursing Studies, 50, 776-785.

30. Gauci, K., Lawnm S., Towns, S., Steinbeck, K. 2021. Effectiveness of self-management programmes for adolescents with a chronic illness: A systematic review. Journal of Advanced Nursing, 77, 3585-3599.

31. Da-Costa, A.C., Rodrigues, F.S., de Fonte, P.P., Rosenblatt, A., Innes, N.P.T., Heimer, M.V. 2017. Influence of sense of coherence on adolescents' self-perceived dental aesthetics; a cross-sectional study. BMC Oral Health, 17(1), 117.

32. Wainwright, N.W., Surtees, P.G., Welch, A.A., Luben, R.N., Khaw, K.T., Bingham, S.A. 2007. Healthy lifestyle choices: Could sense of coherence aid health promotion?. Journal Epidemiol Community Health, 61(10), 871-876.

33. Guner, U.C., Celebioglu, A. 2015. Impact of symptom management training among asthmatic children and adolescents of selfefficacy and disease course. Journal of Asthma, 52(8), 858-865.

34. Janssens, T., Harver, A. 2015. Effects of symptom perception interventions on trigger identification and quality of life in children with asthma. Pulmonary Medicine, 825137.

35. Lloyd, S., Chalder, T., Rimes, K.A. 2012. Family-focused cognitive behaviour therapy versus psycho-education for adolescents with chronic fatigue syndrome: long-term follow-up of an RCT. Behav Res Ther, 50(11), 719-725.

36. Quinn, G.P., Gonçalves, V., Sehovic, I., Bowman, M.L., Reed, D.R. (2015). Quality of life in adolescent and young adult cancer patients: A systematic review of the literature. Patient Related Outcome Measures, 6, 19-51.

37. Carona, C., Moreira, H., Silva, N., Crespo, C., Canavarro, M.C. 2014. Social support and adaptation outcomes in children and adolescents with cerebral palsy. Disability and Rehabilitation, 36, 584-92.

38. Nordang, K, Hall-Lord, M.L, Farup, P.G. (2010) Burnout in health-care professionals during reorganizations and downsizing. A cohort study in nurses. BMC Nursing, 9, 1-7.

39. Richardson, C., Ratner, P. 2005. Sense of coherence as a moderator of the effects of stressful live events on health. Journal of Epidemiology \& Community Health, 59, 979-984.

40. Antonovsky, A. 1996. The salutogenic model as a theory to guide health promotion. Health Promotion International, 11, 1118.

41. Lundman, B., Alex, L., Jonsen, E., Norberg, A., Nygren, B., Fischer, R.S., Strandberg, G. 2010. Inner strength A theoretical analysis of salutogenic concepts. International Journal of Nursing Studies, 47(2), 251-260.

\section{Tables}

Table 1. Participant Characteristics $(\mathrm{N}=239)$ 


\begin{tabular}{|c|c|c|}
\hline Variables & Categories & $\begin{array}{l}\text { Mean } \pm S D \text { ol } \\
\mathrm{n}(\%)\end{array}$ \\
\hline \multirow[t]{2}{*}{ Gender } & Male & 117(49.0) \\
\hline & Female & $122(51.0)$ \\
\hline \multirow[t]{2}{*}{ Age (years) } & $13 \sim 15$ & 167(69.9) \\
\hline & $16 \sim 18$ & $72(30.1)$ \\
\hline \multirow[t]{5}{*}{ Confidence of self-management } & Can't do it at all & $3(1.3)$ \\
\hline & Can do well & $20(8.4)$ \\
\hline & Usually & $132(55.2)$ \\
\hline & Can do well & $64(26.8)$ \\
\hline & Can do very well & $20(8.4)$ \\
\hline \multirow[t]{3}{*}{ Diagnosed path* } & Clinical symptom & 224(93.7) \\
\hline & Medical examination & $6(2.5)$ \\
\hline & $\begin{array}{l}\text { Detected during examination of another } \\
\text { disease }\end{array}$ & $9(3.8)$ \\
\hline \multirow[t]{3}{*}{ Period since diagnosis (months) } & $0 \sim 12$ & $40(16.7)$ \\
\hline & $13-36$ & $76(31.8)$ \\
\hline & $\geq 37$ & $123(51.5)$ \\
\hline \multirow{2}{*}{$\begin{array}{l}\text { Experience in receiving disease information and disease } \\
\text { education }\end{array}$} & Yes & $50(20.9)$ \\
\hline & No & 189(79.1) \\
\hline
\end{tabular}

Table 2. Descriptive statistics and confirmatory factor analyses of measured variables. $(\mathrm{N}=239)$ 


\begin{tabular}{|c|c|c|c|c|c|}
\hline Variables & number of question & $\begin{array}{l}\text { Mean } \\
( \pm S D)\end{array}$ & Range & Skewness & Kurtosis \\
\hline \multirow[t]{2}{*}{ Social support } & 36 & 163.60 & $65 \sim 216$ & -1.03 & -1.69 \\
\hline & & $( \pm 32.11)$ & & & \\
\hline \multirow[t]{2}{*}{ Parent (1-12) } & 12 & 54.42 & $14 \sim 72$ & -2.76 & -1.79 \\
\hline & & $( \pm 12.65)$ & & & \\
\hline Teacher (13-24) + & 24 & 109.18 & $51 \sim 144$ & -1.74 & -1.78 \\
\hline Close friend (25-36) & & $( \pm 22.76)$ & & & \\
\hline \multirow[t]{2}{*}{ Sense of Coherence } & 13 & 61.78 & $27 \sim 90$ & 1.77 & -1.64 \\
\hline & & $( \pm 12.04)$ & & & \\
\hline \multirow[t]{2}{*}{ Comprehensibility $(2,6,8,9,11)$} & 5 & 23.31 & $11 \sim 35$ & 1.67 & -1.87 \\
\hline & & $( \pm 5.21)$ & & & \\
\hline \multirow[t]{2}{*}{ Manageability $(3,5,10,13)$} & 4 & 19.35 & $8 \sim 28$ & 0.80 & -1.87 \\
\hline & & $( \pm 4.17)$ & & & \\
\hline \multirow[t]{2}{*}{ Meaningfulness $(1,4,7,12)$} & 4 & 19.12 & $6 \sim 28$ & 0.50 & -0.22 \\
\hline & & $( \pm 4.06)$ & & & \\
\hline \multirow[t]{2}{*}{ Stress } & 27 & 43.71 & $16 \sim 103$ & 8.48 & 5.35 \\
\hline & & $( \pm 14.98)$ & & & \\
\hline \multirow[t]{2}{*}{ Physical(15-27) } & 13 & 20.42 & $0 \sim 51$ & 9.03 & 7.87 \\
\hline & & $( \pm 7.46)$ & & & \\
\hline \multirow[t]{2}{*}{ Behavior(1-5,8) } & 6 & 10.9 & $6 \sim 23$ & 6.10 & 2.32 \\
\hline & & $3( \pm 3.88)$ & & & \\
\hline \multirow[t]{2}{*}{ Emotion(6,7,9-14) } & 8 & 12.36 & $6 \sim 32$ & 11.04 & 9.55 \\
\hline & & $( \pm 5.18)$ & & & \\
\hline \multirow[t]{2}{*}{ Health behavior } & 12 & 41.13 & $15 \sim 60$ & -1.21 & -0.64 \\
\hline & & $( \pm 9.19)$ & & & \\
\hline Treatment instructions & 12 & 41.13 & $15 \sim 60$ & -1.21 & -0.64 \\
\hline$(1-4)+$ & & $( \pm 9.19)$ & & & \\
\hline \multicolumn{6}{|l|}{ Health coping behavior } \\
\hline \multicolumn{6}{|l|}{$(5-8)+$} \\
\hline \multicolumn{6}{|l|}{ Health promotion acts } \\
\hline \multicolumn{6}{|l|}{$(9-12)$} \\
\hline \multirow[t]{2}{*}{ Quality of Life } & 23 & 92.43 & $45 \sim 115$ & -4.00 & 1.28 \\
\hline & & $( \pm 14.99)$ & & & \\
\hline \multirow[t]{2}{*}{ Physical Function (1-8) } & 8 & 31.43 & $13 \sim 40$ & -2.82 & -1.18 \\
\hline & & $( \pm 5.82)$ & & & \\
\hline \multirow[t]{2}{*}{ Emotional function (9-13) } & 5 & 20.0 & $5 \sim 25$ & -5.13 & 0.61 \\
\hline & \multicolumn{2}{|c|}{ Page $13 / 18$} & & & \\
\hline
\end{tabular}


Interpersonal skills $(14-18)+10$

School function (19-23)
40.91

Table3. Correlation among the observed variables

\begin{tabular}{|c|c|c|c|c|c|c|c|c|c|c|c|}
\hline & $\mathrm{x} 1$ & $x 2$ & $x 3$ & $x 4$ & $x 5$ & $\mathrm{y} 1$ & y2 & y3 & y6 & y7 & y8 \\
\hline$x 1$ & 1.000 & & & & & & & & & & \\
\hline$x 2$ & $0.614^{\star \star \star}$ & 1.000 & & & & & & & & & \\
\hline$x 3$ & $0.451^{\star \star \star}$ & $0.509^{\star \star \star}$ & 1.000 & & & & & & & & \\
\hline$x 4$ & $0.461^{\star \star \star}$ & $0.474^{\star \star \star}$ & $0.751^{\star \star \star}$ & 1.000 & & & & & & & \\
\hline$x 5$ & $0.489^{\star \star \star}$ & $0.583^{\star \star \star}$ & $0.705^{\star \star \star}$ & $0.630^{\star \star \star}$ & 1.000 & & & & & & \\
\hline y1 & $-0.147^{\star}$ & -0.098 & $-0.229^{\star \star}$ & $-0.172^{\star \star}$ & $-0.205^{\star \star}$ & 1.000 & & & & & \\
\hline y2 & -0.119 & $-0.172^{\star *}$ & $-0.207^{\star \star}$ & $-0.167^{*}$ & $-0.206^{\star \star}$ & $0.671^{\star \star \star}$ & 1.000 & & & & \\
\hline y3 & -0.097 & $-0.218^{\star \star}$ & $-0.303^{\star \star \star}$ & $-0.230^{\star \star \star}$ & $-0.286^{\star \star \star}$ & $0.764^{\star \star \star}$ & $0.719^{\star * \star}$ & 1.000 & & & \\
\hline y6 & $0.174^{\star \star}$ & $0.274^{\star \star \star}$ & $0.414^{\star \star \star}$ & $0.282^{\star \star \star}$ & $0.463^{\star \star \star}$ & $-0.387^{\star \star \star}$ & $-0.387^{\star \star \star}$ & $-0.443^{\star \star \star}$ & 1.000 & & \\
\hline y7 & $0.196^{\star \star}$ & $0.375^{\star \star \star}$ & $0.511^{\star \star \star}$ & $0.399^{\star \star \star}$ & $0.458^{\star \star \star}$ & $-0.452^{\star \star}$ & $-0.583^{\star \star \star}$ & $-0.659^{\star \star \star}$ & $0.639^{\star \star \star}$ & 1.000 & \\
\hline y8 & $0.293^{\star \star \star}$ & $0.506^{\star \star \star}$ & $0.425^{\star \star \star}$ & $0.376^{\star \star \star}$ & $0.481^{\star \star \star}$ & $-0.268^{\star * \star}$ & $-0.312^{\star \star \star}$ & $-0.428^{\star \star \star}$ & $0.683^{\star \star \star}$ & $0.626^{\star \star *}$ & 1.000 \\
\hline
\end{tabular}

$p<0.05:{ }^{*}, p<0.01: * \star, p<0.001: * \star *$

$x 1=$ Social support(Parent), $x 2=$ Social support(Teacher+Close friend), $x 3=$ Sense of Coherence(Meaningfulness), $x 4=$ Sense of Coherence(Manageability), x5= Sense of Coherence(Comprehensibility), y1 = Stress(Behavior), y2=Stress(Physical), y3=Stress(Emotion), y6=Quality of Life(Physical), y7= Quality of Life(Emotional), y8= Quality of Life(Interpersonal+School)

Table 4. Model Fit of the hypothetical Model $(n=239)$

\begin{tabular}{lllllllllll} 
Model & $\mathbf{x}^{2}(\boldsymbol{p})$ & $\mathrm{DF}$ & $\mathbf{x}^{2} / \mathbf{d f}$ & $\mathrm{GFI}$ & $\mathrm{AGFI}$ & $\mathrm{CFI}$ & $\mathrm{NFI}$ & IFI & SRMR & RMSEA \\
\hline Reference & $(.05)$ & & $<3$ & $.90-1$ & $.90-1$ & $.90-1$ & $.90-1$ & $.90-1$ & $\leq .05$ & $\leq .08$ \\
\hline Initial model & 469.359 & 105 & 4.470 & 0.814 & 0.729 & 0.854 & 0.821 & 0.855 & 0.075 & 0.121 \\
& $(p<.001)$ & & & & & & & & & \\
\hline Final model & 211.446 & 52 & 4.066 & 0.887 & 0.803 & 0.910 & 0.885 & 0.911 & 0.052 & 0.114 \\
& $(p<.001)$ & & & & & & & & &
\end{tabular}

GFI, goodness of fit index; AGFI, adjusted goodness-to-fit index; NFI, normed fit index; IFI, incremental fit index; SRMR, standardized root mean squared residual; RMSEA, root mean square error of approximation; CFI, comparative fit index

Table 5. Standardized Estimates for Hypothetical Model 


\begin{tabular}{|c|c|c|c|c|c|c|}
\hline $\begin{array}{l}\text { Endogeneous } \\
\text { Variables }\end{array}$ & Explanatory variable & Estimate & $\begin{array}{l}\text { Standardized } \\
\text { estimates }(\beta)\end{array}$ & S.E. & C.R.(p) ${ }^{\dagger}$ & $\operatorname{SMC}(\mathrm{R} 2)^{\ddagger}$ \\
\hline \multirow[t]{2}{*}{ Stress } & Social Support & 0.034 & 0.052 & 0.085 & 0.402 & \multirow[t]{2}{*}{0.113} \\
\hline & Sense of Coherence & -0.684 & -0.373 & 0.231 & $-2.958 \star \star$ & \\
\hline \multirow[t]{3}{*}{ Health Behavior } & Social Support & 0.321 & 0.325 & 0.115 & $2.788^{\star \star}$ & \multirow[t]{3}{*}{0.274} \\
\hline & Sense of Coherence & 0.494 & 0.178 & 0.317 & 1.560 & \\
\hline & Stress & -0.198 & -0.131 & 0.097 & $-2.038 *$ & \\
\hline \multirow[t]{4}{*}{ Subjective Health Status } & Health Behavior & 0.003 & 0.032 & 0.006 & 0.474 & \multirow[t]{4}{*}{0.295} \\
\hline & Stress & 0.002 & 0.015 & 0.008 & 0.242 & \\
\hline & Social Support & 0.007 & 0.084 & 0.010 & 0.723 & \\
\hline & Sense of Coherence & 0.112 & 0.467 & 0.027 & $4.146^{\star \star \star}$ & \\
\hline \multirow[t]{5}{*}{ Quality of Life } & Subjective Health Status & 0.613 & 0.108 & 0.331 & 1.855 & \multirow[t]{5}{*}{0.689} \\
\hline & Health Behavior & -0.085 & -0.173 & 0.029 & $-2.968 * \star$ & \\
\hline & Stress & -0.414 & -0.557 & 0.049 & $-8.399 * \star \star$ & \\
\hline & Social Support & 0.066 & 0.137 & 0.048 & 1.370 & \\
\hline & Sense of Coherence & 0.504 & 0.371 & 0.141 & $3.575^{\star \star \star}$ & \\
\hline
\end{tabular}

${ }^{\star} p<.05, * \star p<0.01, * \star \star x<.001$ ( ${ }^{\dagger}$ Critical Ratio; ${ }^{\ddagger}$ Squared Multiple Correlations)

Table 6. Standardized Direct, Indirect and Total Effects of the Modified Model $(n=239)$ 


\begin{tabular}{|c|c|c|c|c|c|}
\hline \multirow[t]{2}{*}{ Endogenous variable } & \multirow[t]{2}{*}{ Exogenous variable } & Standardized & Standardized & Standardized & \multirow[t]{2}{*}{$\operatorname{SMC}\left(R^{2}\right)^{\dagger}$} \\
\hline & & Direct Effects & Indirect Effects & Total Effects & \\
\hline \multirow[t]{2}{*}{ Stress } & Social Support & $0.052(0.687)$ & - & $0.052(0.687)$ & 0.113 \\
\hline & $\mathrm{SOC}^{\square}$ & $0.373\left(0.004^{\star \star}\right)$ & - & $-0.373(0.004 \star \star)$ & \\
\hline \multirow[t]{3}{*}{ Health Behavior } & Social Support & $0.325\left(0.006^{\star \star}\right)$ & $-0.007(0.568)$ & $0.318\left(0.005^{\star \star}\right)$ & 0.274 \\
\hline & $\mathrm{SOC}^{\square}$ & $0.178(0.165)$ & $0.049\left(0.027^{\star}\right)$ & $0.227(0.050)$ & \\
\hline & Stress & $-0.131\left(0.043^{\star}\right)$ & - & $-0.131\left(0.043^{\star}\right)$ & \\
\hline \multirow[t]{6}{*}{ Subjective health Status } & Health Behavior & $0.032(0.628)$ & - & $0.032(0.628)$ & 0.295 \\
\hline & Stress & & & & \\
\hline & Social Support & $0.015(0.828)$ & $-0.004(0.487)$ & $0.011(0.879)$ & \\
\hline & $\mathrm{SOC}^{\square}$ & & & & \\
\hline & & $0.084(0.486)$ & $0.011(0.567)$ & $0.094(0.441)$ & \\
\hline & & $0.467(<.001 * \star \star)$ & $0.001(0.879)$ & $0.468\left(<.001^{\star \star \star}\right)$ & \\
\hline \multirow[t]{7}{*}{ Quality of Life } & Subjective Health Status & $0.108(0.056)$ & - & $0.108(0.056)$ & 0.689 \\
\hline & Health Behavior & & & & \\
\hline & Stress & $-0.173(0.009 * \star)$ & $0.003(0.450)$ & $-0.169\left(0.010^{\star}\right)$ & \\
\hline & Social Support & & & & \\
\hline & $\mathrm{SOC}^{\square}$ & $0.557\left(<.001^{\star \star \star}\right)$ & $0.024(0.053)$ & $-0.534\left(<.001^{\star \star *}\right)$ & \\
\hline & & $0.137(0.271)$ & $-0.074(0.339)$ & $0.063(0.689)$ & \\
\hline & & $0.371\left(0.003^{\star \star}\right)$ & $0.220\left(0.013^{\star}\right)$ & $0.590\left(<.001^{\star \star \star}\right)$ & \\
\hline
\end{tabular}

${ }^{*} p<.05,{ }^{* *} p<0.01,{ }^{* * *} p<.001\left({ }^{\dagger}\right.$ Squared Multiple Correlations, ${ }^{\square}$ Sense of Coherence)

\section{Figures}




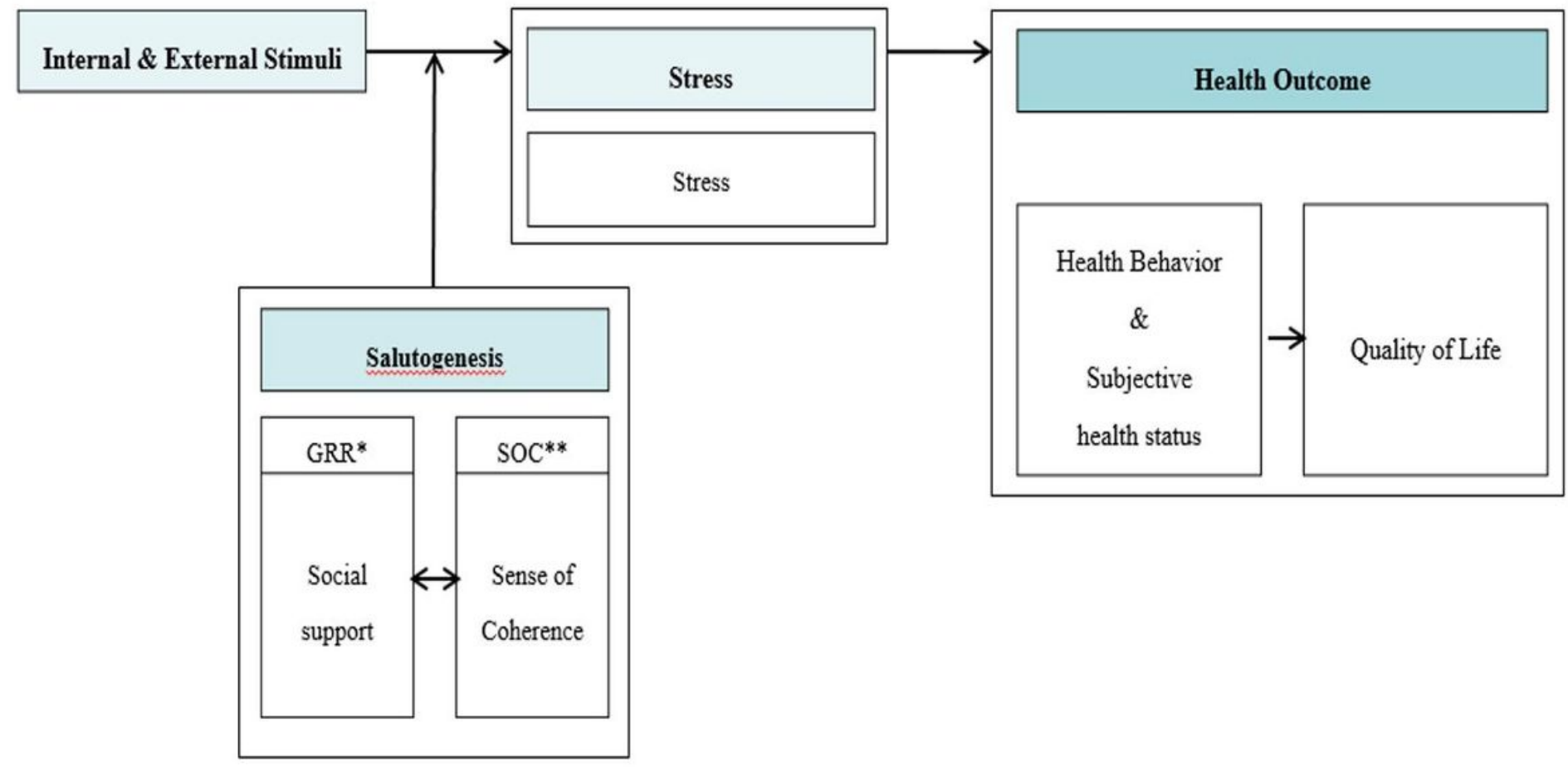

\section{Figure 1}

Theoretical framework based on the salutogenesis theory.

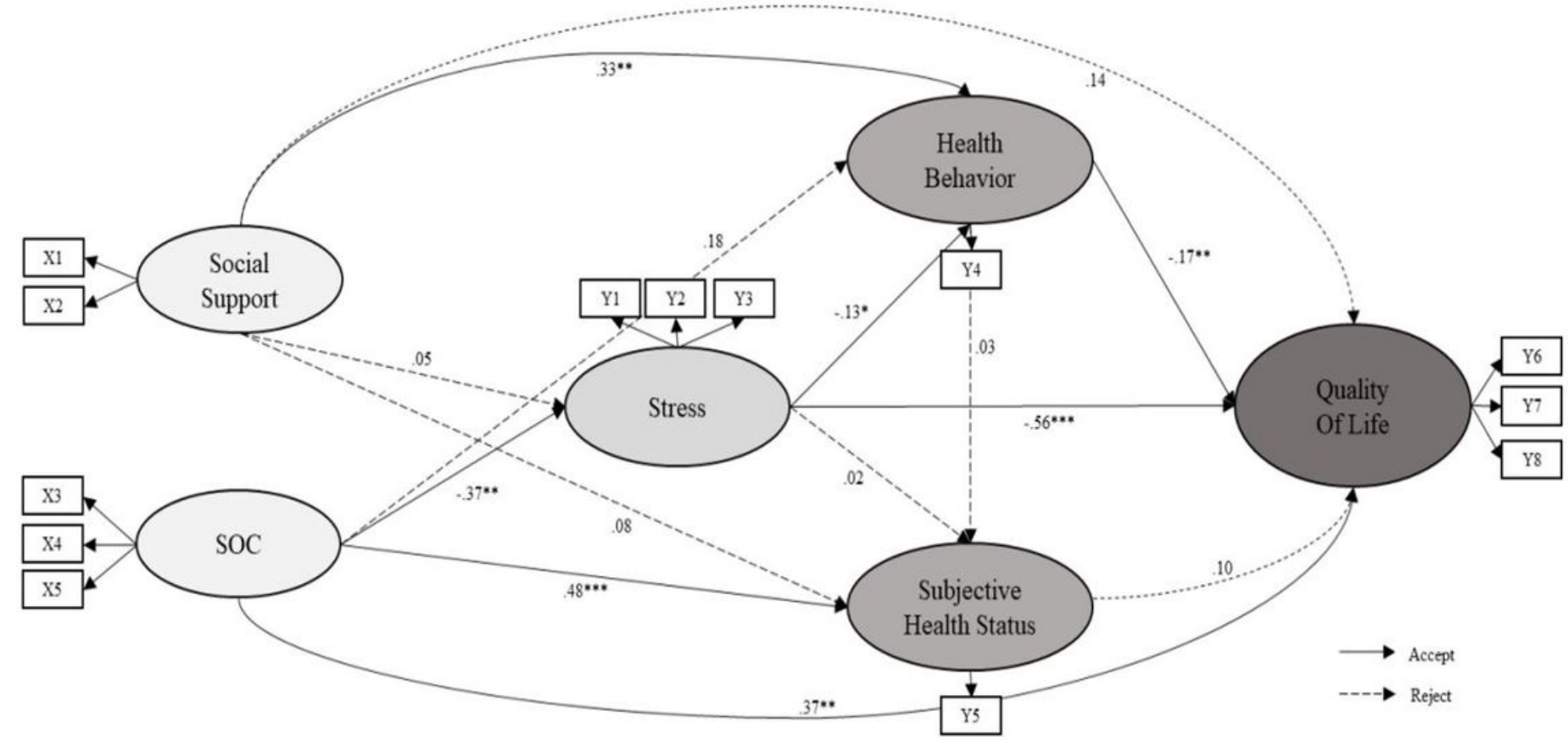

Figure 2

Path diagram for the hypothetical structural equation model. $x 1=$ Social support (Parent), $x 2=$ Social support (Teacher+Close friend), $x 3$ = Sense of Coherence (Meaningfulness), $x 4$ = Sense of Coherence (Manageability), x5 = Sense of Coherence (Comprehensibility), y1 = Stress (Behavior), y2 = Stress (Physical), y3 = Stress (Emotion), y4 = Healthy behavior (Treatment Instructions, Health coping behavior, Health Promotion Acts), y5 = self-management confidence, y6 = Quality of life (physical), y7 = Quality of life (emotional), y8 = Quality of life (interpersonal relationship + school) 


\section{Supplementary Files}

This is a list of supplementary files associated with this preprint. Click to download.

- STROBEchecklistofcrosssectionalstudies.docx 